ournal for ImmunoTherapy of Cancer

\section{Phase 1 open-label trial of intravenous administration of MVA-BN-brachyury- TRICOM vaccine in patients with advanced cancer}

To cite: DeMaria PJ, LeeWisdom K, Donahue RN, et al. Phase 1 open-label trial of intravenous administration of MVA-BN-brachyury-TRICOM vaccine in patients with advanced cancer. Journal for ImmunoTherapy of Cancer 2021;9:e003238. doi:10.1136/ jitc-2021-003238

- Additional supplemental material is published online only. To view, please visit the journal online (http://dx.doi.org/10. 1136/jitc-2021-003238).

Accepted 08 July 2021

Check for updates

(C) Author(s) (or their employer(s)) 2021. Re-use permitted under CC BY-NC. No commercial re-use. See rights and permissions. Published by BMJ.

For numbered affiliations see end of article.

Correspondence to

Dr Marijo Bilusic;

marijo.bilusic@nih.gov

\section{ABSTRACT}

Background MVA-BN-brachyury-TRICOM is a recombinant vector-based therapeutic cancer vaccine designed to induce an immune response against brachyury. Brachyury, a transcription factor overexpressed in advanced cancers, has been associated with treatment resistance, epithelial-to-mesenchymal transition, and metastatic potential. MVA-BN-brachyury-TRICOM has demonstrated immunogenicity and safety in previous clinical trials of subcutaneously administered vaccine. Preclinical studies have suggested that intravenous administration of therapeutic vaccines can induce superior $\mathrm{CD}^{+} \mathrm{T}$ cell responses, higher levels of systemic cytokine release, and stronger natural killer cell activation and proliferation. This is the first-in-human study of the intravenous administration of MVA-BN-brachyury-TRICOM. Methods Between January 2020 and March 2021, 13 patients were treated on a phase 1, open-label, $3+3$ design, dose-escalation study at the National Institutes of Health Clinical Center. The study population was adults with advanced solid tumors and was enriched for chordoma, a rare sarcoma of the notochord that overexpresses brachyury. Vaccine was administered intravenously at three DLs on days 1, 22, and 43. Blood samples were taken to assess drug pharmacokinetics and immune activation. Imaging was conducted at baseline, 1 month, and 3 months post-treatment. The primary endpoint was safety and tolerability as determined by the frequency of dose-limiting toxicities; a secondary endpoint was determination of the recommended phase 2 dose. Results No dose-limiting toxicities were observed and no serious adverse events were attributed to the vaccine. Vaccine-related toxicities were consistent with class profile (ie, influenza-like symptoms). Cytokine release syndrome up to grade 2 was observed with no adverse outcomes. Dose-effect trend was observed for fever, chills/rigor, and hypotension. Efficacy analysis of objective response rate per RECIST 1.1 at the end of study showed one patient with a partial response, four with stable disease, and eight with progressive disease. Three patients with stable disease experienced clinical benefit in the form of improvement in pain. Immune correlatives showed T cell activation against brachyury and other tumor-associated cascade antigens.

Conclusions Intravenous administration of MVA-BNbrachyury-TRICOM vaccine was safe and tolerable. Maximum tolerated dose was not reached. The maximum administered dose was $10^{9}$ infectious units every 3 weeks for three doses. This dose was selected as the recommended phase 2 dose.

Trial registration number NCT04134312.

\section{INTRODUCTION}

MVA-BN-brachyury-TRICOM is a recombinant vector-based therapeutic cancer vaccine. The vector is modified vaccinia Ankara (MVA) virus, designed to induce an enhanced immune response against brachyury, which is overexpressed in many solid tumors such as lung, breast, ovarian, chordoma, prostate, colorectal, and pancreatic adenocarcinoma. ${ }^{1}$ Brachyury is a member of the T-box family of transcription factors, characterized by a highly conserved DNA-binding domain designated as the T-domain. ${ }^{2-5}$ The gene encoding for the transcription factor brachyury was identified using a computer-based differential display analysis tool to conduct global comparison of expressed sequence tag clusters in the Unigene database. ${ }^{6}$ Brachyury homologs are involved in embryonic mesodermal development and play a vital role during early embryonic gastrulation and notochord formation. ${ }^{2}$ 7-10 After embryonic development, brachyury is expressed in the testes, nucleus pulposus cells, and thyroid tissues but is undetectable in all other healthy adult tissues. $^{361112}$ In cancer, brachyury is thought to be a driver of epithelial-to-mesenchymal 
transition, a reversible process during which cells switch from a polarized, epithelial phenotype into a highly motile, mesenchymal phenotype. ${ }^{13}$ Epithelial-to- $^{14}$ mesenchymal transition involves the downregulation of epithelial proteins such as E-cadherin and cytokeratins and the induction of mesenchymal proteins including fibronectin, N-cadherin, and vimentin. ${ }^{15}{ }^{16}$ It has been demonstrated that overexpression of brachyury in human carcinoma cell lines drives a switch from an epithelial to a mesenchymal-like phenotype. ${ }^{17}$ Overexpression of brachyury in epithelial tumor cells results in a concomitant increase in tumor-cell migration and extracellular matrix invasion. In preclinical studies, brachyury-silenced cells implanted subcutaneously in athymic mice had a diminished capacity to metastasize to the lungs from the primary subcutaneous tumor and showed a reduced ability to form experimental lung metastasis after intravenous implantation. ${ }^{17}$ These results demonstrate that the transcription factor brachyury confers on tumor cells a mesenchymal phenotype as well as migratory and invasive capabilities and enhances tumor-cell progression.

Initial reports of brachyury overexpression in cancer originated from studies in chordoma, a rare sarcoma derived from remnants of the primitive notochord. Chordomas universally overexpress brachyury, ${ }^{18} 19$ while brachyury gene duplication seems to be a major susceptibility factor for familial chordoma. ${ }^{20}$ Brachyury is an essential transcriptional regulator of chordoma identity and is related to super-enhancer functionality, thus representing a therapeutic target of special interest for chordoma. ${ }^{21} 22$ Overexpression of brachyury messenger RNA was also found in gastrointestinal, bladder, kidney, ovarian, uterine, and testicular carcinomas, as well as in cell lines of lung, colon, and prostate cancers. ${ }^{6}$ In contrast, brachyury messenger RNA was notably absent in the vast majority of normal tissues tested, making brachyury an attractive target for the development of novel anticancer therapies.

To date, five investigational therapeutic cancer vaccines targeting brachyury have been studied or remain under investigation in human clinical trials. The first vaccine to undergo human testing was GI-6301 (NCT01519817), a yeast-brachyury vaccine composed of heat-killed recombinant Saccharomyces cerevisiae expressing brachyury. ${ }^{23} \mathrm{~A}$ phase 2 study (NCT02383498) of the S. cerevisiae yeastbrachyury vaccine in combination with radiation therapy in chordoma did not meet its primary efficacy endpoint. ${ }^{24}$ The next vaccine to undergo human trials was MVAbrachyury-TRICOM, an MVA vector-based vaccine expressing the transgenes for brachyury and three human T cell costimulatory molecules: B7.1, ICAM-1, and LFA-3, designated as TRICOM. After the phase 1 dose-finding trial (NCT02179515), a second phase 1 study (NCT03349983) employed a heterologous 'prime and boost' strategy with MVA-brachyury-TRICOM as the priming vaccine and fowlpox virus (FPV)-brachyury-TRICOM (FPV-brachyury) as the booster vaccine. ${ }^{25} 26$ The Bavarian Nordic (BN)brachyury therapeutic cancer vaccine (consisting of the 'prime and boost' regimen) is currently under investigation in a phase 2 study in combination with radiation therapy in chordoma (NCT03595228) and in combination with other systemic therapies in breast and prostate cancer (NCT03493945, NCT04020094, NCT04296942). Clinical trials of adenovirus-vector vaccines (ie, ETBX-051) targeting brachyury are also underway (NCT03384316, NCT03481816).

Previous clinical trials of therapeutic cancer vaccines have generally deployed the vaccine product via subcutaneous or intramuscular routes. Preclinical studies have demonstrated that intravenous administration of some vaccines, including MVA-based constructs, can induce superior immunogenicity compared with subcutaneous or intramuscular routes. A preclinical study comparing different routes of administration of an MVA-vectored HIV vaccine found that the intravenous route was more efficient in inducing $\mathrm{T}$ cell immunity. In addition, the analysis of systemic cytokine responses demonstrated a strong production of monocyte chemoattractant protein 1 and 3 (MCP-1 and MCP-3), regulated upon activation, normal $\mathrm{T}$ cell expressed and secreted chemokine (RANTES), interleukin (IL)-6, IL-18, and interferon (IFN) $-\gamma$ following intravenous administration. Consistent with cytokine production, stronger NK and T cell activation was associated with intravenous administration. ${ }^{27-29}$ Here, we present results from the first-in-human clinical trial of an intravenously administered therapeutic cancer vaccine targeting brachyury. NCT04134312 was a singlesite, open-label, phase 1, dose-escalation study of intravenously administered MVA-BN-brachyury-TRICOM. The investigational biologic product, also known as MVA-mBN240B, is a liquid-frozen, highly attenuated, live recombinant virus that was supplied by the study sponsor, Bavarian Nordic. The primary objectives of this trial were to assess the safety and tolerability of the vaccine and to determine the recommended phase 2 dose (RP2D).

\section{MATERIALS AND METHODS Patient eligibility}

The study population was adults with advanced solid tumors and was enriched for chordoma. Eligible histologies included tumors with known overexpression of brachyury such as non-small cell lung, small cell lung, breast, ovarian, prostate, colorectal, pancreas, hepatocellular, bladder, and kidney carcinomas. Eligible patients were required to have metastatic or incurable disease, with disease progression or intolerance to systemic therapies with known benefit; specific treatment and the number of lines of prior treatment required to enroll varied by tumor type. Patients were required to have measurable disease per Response Evaluation Criteria in Solid Tumors (RECIST) 1.1 or evaluable disease defined by the presence of elevated serum tumor markers and/ or clear radiographic or clinical evidence of tumor which did not meet RECIST 1.1 measurement requirements. ${ }^{30}$ Enrollment was limited to patients with an Eastern 
Cooperative Oncology Group (ECOG) performance status of 0 or 1 at study entry. Normal organ and bone marrow function were required with standard parameters. Women of childbearing potential were required to have a negative pregnancy test within 48 hours of treatment; breast feeding was not permitted. Men and women of childbearing potential agreed to use effective birth control or abstinence during the study and for 3 months after the last vaccination. Patients were required to have completed any prior treatment at least 4 weeks prior to the first planned dose of vaccine, the exception being 6 weeks for any prior antibody therapies due to prolonged half-life. Patients must have recovered to either grade 1 or baseline from any clinically significant toxicity associated with prior therapy. The study did not enroll patients with serious intercurrent illness, active infection, recent administration of antibiotics, active autoimmune disease, immunosuppression, HIV, chronic active hepatitis, or hypersensitivity to any vaccine, aminoglycoside, or egg product. Chronic systemic steroid use was prohibited within 4 weeks of study enrollment; physiologic replacement dosing was permitted.

\section{Study design}

The study explored three dose levels (DL) of vaccine: $1 \times 10^{7}$ infectious units (Inf. U.) (DL 1$), 1 \times 10^{8}$ Inf. U. (DL 2), and $1 \times 10^{9}$ Inf. U. (DL 3). Based on preclinical data, we anticipated that the change in the route of administration from subcutaneous (SC) to intravenous would speed the dynamics of cytokine release with potential increase of the cytokine levels in blood. Concern for potential cytokine release syndrome (CRS) was the rationale for proceeding with a dose-escalation phase 1 design up to $1 \times 10^{9}$ Inf. U., which has been historically the maximum dose administered SC. Following a screening period and informed consent, patients were admitted on day -1 to the inpatient service at the National Institutes of Health (NIH) Clinical Center in Bethesda, Maryland, USA. Three intravenous treatments were administered every 3 weeks on days 1, 22, and 43. Blood samples were taken at prespecified time points to assess drug pharmacokinetics and immune activation. Inpatient hospitalization was necessary for pharmacokinetic assessment and patient safety due to the potential for immune activation and CRS. Patients were discharged 2 days post-treatment on days 3,24 , and 45 ; they returned 1 week after each treatment for a safety check consisting of complete history and physical with laboratory assessment and ECG. Imaging with formal response assessment per RECIST 1.1 was conducted at baseline, 1 month, and 3 months post-final vaccination. Trial duration was approximately 24 weeks per patient, including a screening period of approximately 4 weeks, 8 weeks of active inpatient treatment, and 3 months of follow-up (figure 1).

The primary endpoint was safety and tolerability as determined by the frequency of dose-limiting toxicities (DLTs). Adverse events (AEs) were assessed by the investigator and graded per the National Cancer Institute's

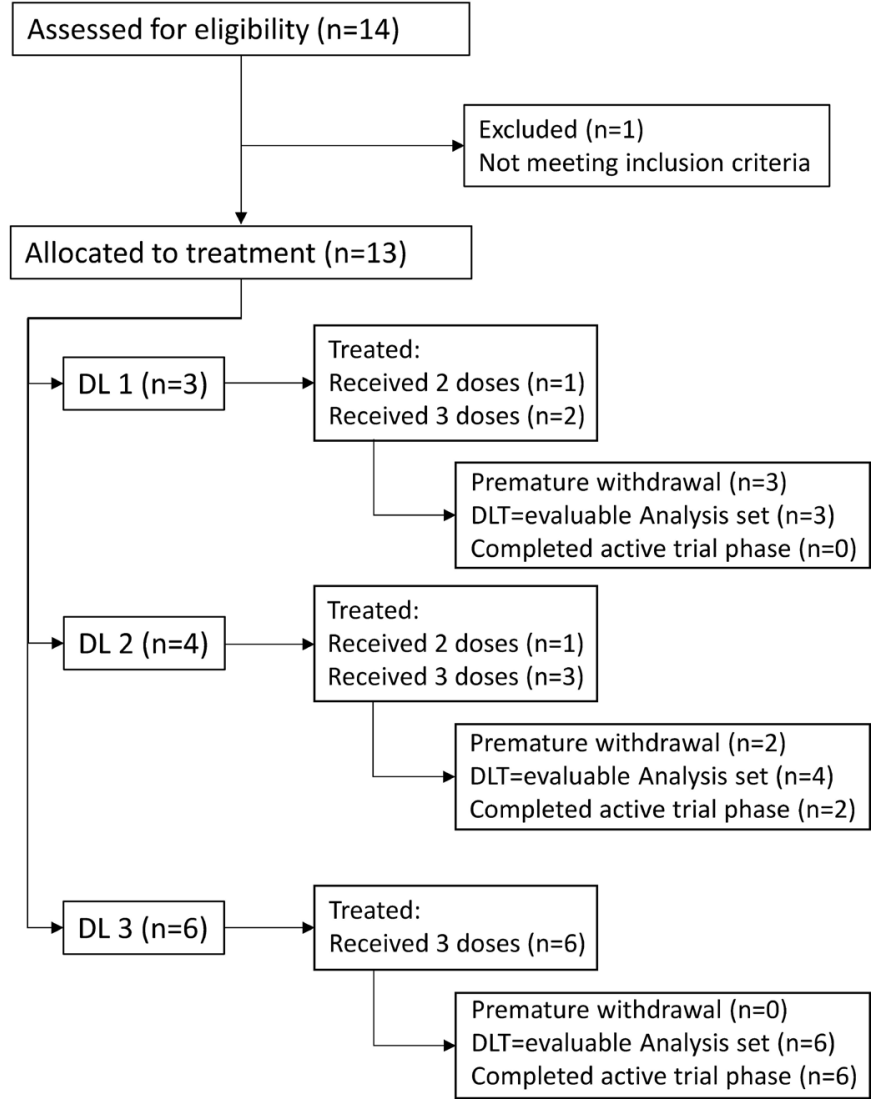

Figure 1 Consort flow diagram. Of 14 patients officially screened for the trial, only one patient was unable to obtain imaging with MRI to confirm eligibility. The other 13 patients screened were treated ( 3 at DL1, 4 at DL2 and 6 at DL3). One patient (patient \#7) treated at DL2 was replaced for failure to complete the DLT period due to treatment delay associated with the SARS-CoV-2 pandemic. DL, dose level; DLT, doselimiting toxicity.

(NCI's) Common Terminology Criteria for Adverse Events V.5.0, ${ }^{31}$ except CRS which was graded as per American Society for Blood and Marrow Transplantation CRS Consesus grading. ${ }^{32}$ Dose escalation followed typical $3+3$ design principles. The maximum tolerated dose was defined as the dose at which the probability of a DLT was $\leq 16.7 \%$. Patient treatment was staggered for safety, which was monitored jointly by the research team, the NIH's Institutional Review Board, and the study sponsor. Dose escalation could not proceed without the agreement of the safety monitoring team. Stopping rules for toxicity were enumerated for individual patients and the overall study. Patients who dropped out of the study prior to receiving the second vaccination, and who had no DLTs, were replaced. No intrapatient dose escalation was permitted.

\section{Statistical and data analyses}

The trial was not powered to test any hypothesis and no formal inferential statistical analysis was performed. Exploratory assessments of immunogenicity included measurement of $\mathrm{T}$ cell responses to tumor-associated antigens (TAAs). Exploratory efficacy analysis included 
assessment of tumor response by objective response rate (ORR) per RECIST 1.1, 3 months after the final vaccination. Exploratory analysis using volumetric tumor segmentation was performed in patients with chordoma to assess tumor response more sensitively using Medx software (Medical Numerics, Germantown, Maryland, USA). Chordoma volume was measured by manually outlining the lesions on MRI or CT images and multiplying the area within the contours by the image slice thickness. Pain scores and daily analgesic requirements were self reported and no standardized Quality of life (QOL) questionare was used. All data reported here are collected up to April 14, 2021.

\section{Immune assays}

Research blood samples for measurement of peripheral $\mathrm{T}$ cell responses to TAA were collected at baseline, prior to each vaccine administration, and at the endoftreatment. These studies were performed by the NCI's Laboratory of Tumor Immunology and Biology. Peripheral blood was collected in sodium heparin tubes and peripheral blood mononuclear cells (PBMCs) were separated by FicollHypaque density gradient separation. The resulting cells were cryopreserved in 90\% heat-inactivated human $\mathrm{AB}$ serum and $10 \%$ dimethyl sulfoxide at a concentration of $1 \times 10^{7}$ cells $/ \mathrm{mL}$. Cryopreserved PBMCs were assessed for $\mathrm{T}$ cell responses to brachyury, the target antigen encoded by the vaccine, as well as against carcinoembryonic antigen (CEA) and mucin-1 (MUC1), antigens not encoded by the vaccine. This assay has been previously described and involves stimulating PBMCs with overlapping 15-mer peptide pools and evaluating $\mathrm{CD} 4^{+}$ and $\mathrm{CD}^{+} \mathrm{T}$ cells for the production of cytokines (IFN- $\gamma$, tumor necrosis factor (TNF)- $\alpha$, IL-2) or for expression of a degranulation marker (CD107a) by flow cytometry. ${ }^{33}$ Fluorophore-conjugated antibodies included anti-human CD4 clone OKT4, CD8 clone RPA-T8, IFN- $\gamma$ clone B27, TNF- $\alpha$ clone MAb11, IL-2 clone MQ1-17H12, and CD107a clone H4A3. All peptide pools were purchased from JPT; peptide pools encoding human leukocyte antigen (HLA) and CEFT (a mixture of peptides of cytomegalovirus, Epstein-Barr virus, influenza, and tetanus toxin) were used as negative and positive controls, respectively. For analysis of immune responses, at least $3 \times 10^{5}$ events in the live gate were acquired with a BD LSR Fortessa equipped with a UV, violet, blue, red, and yellow/green laser, and Flow Cytometry Standard (FCS) files were analyzed with FlowJo V.9.9.6 for Macintosh (TreeStar). The absolute number of viable $\mathrm{CD}^{+}$or $\mathrm{CD}^{+} \mathrm{T}$ cells producing cytokine or positive for CD107a at the end of the expansion was calculated per $1 \times 10^{6}$ cells plated at the start of the stimulation assay. This calculation accounts for not only the percentage but also the total number of viable antigen-specific $\mathrm{T}$ cells expanded in the stimulation assay. The background signal (obtained with the HLA peptide pool) and any value obtained prior to treatment were subtracted from those obtained after treatment ((post-TAA-post-HLA)-(pre-TAA-pre-HLA)). A patient was scored as developing a response to a TAA if a patient had more than $250 \mathrm{CD} 4^{+}$or $\mathrm{CD} 8^{+} \mathrm{T}$ cells that produced IFN- $\gamma$, TNF- $\alpha$, or IL-2 or were positive for CD107a at the end of the stimulation assay per $1 \times 10^{6}$ cells that were plated at the start of the assay. Multifunctional $\mathrm{T}$ cells, defined as $\mathrm{CD}^{+}$or $\mathrm{CD}^{+} \mathrm{T}$ cells expressing two or more of IFN- $\gamma$, TNF- $\alpha$, IL-2, or CD107a, were also evaluated. Patients were scored as developing multifunctional T cells during therapy if there was a more than 3-fold increase after versus before vaccination.

To determine the degree of $\mathrm{T}$ cell infiltrate in a biopsy sample obtained 2 days after the third vaccine in patient \#9, tissue was minced, digested using a human tumor dissociation kit (Miltenyi) per manufacturer protocol, and the resulting suspension filtered $(70 \mu \mathrm{m})$. Red blood cells were lysed, and single cell suspensions blocked with fc block (BD Biosciences) prior to staining with antibodies (all purchased from Biolegend) using methods previously described.$^{34}$ Fluorophore-conjugated antibodies included anti-human CD45 clone HI30, CD4 clone OKT4, CD8 clone RPA-T8, CCR7 clone G043H7, and CD45RA clone HI100. A live dead discriminator (live/dead fixable blue, Invitrogen) was included, and 'fluorescence minus one' controls were used for gating.

\section{RESULTS}

\section{Patient population}

Between January 2020 and March 2021, 13 patients were treated at the NIH Clinical Center in Bethesda, Maryland, USA. Mean patient age was 60 (range: 46-77). Male $(69 \%)$ and Caucasian (69\%) patients predominated across all DLs. Patients of color were represented, including two African Americans (15\%), one Asian $(8 \%)$, and one Native American (8\%). Patients had a preserved ECOG performance status of either $0(38.5 \%)$ or $1(61.5 \%)$. The overall cohort included 10 patients (76.9\%) with chordoma, $1(7.7 \%)$ with small cell breast cancer, $1(7.7 \%)$ with colorectal cancer, and $1(7.7 \%)$ with prostate cancer. The patients with breast, colorectal, and prostate cancer were treated on DL1, while DL2 and DL3 were given exclusively to patients with chordoma. While the study was enriched for chordoma, the treatment of non-chordoma patients exclusively at DL1 was not preplanned. At baseline, $46.2 \%$ of all patients had locally advanced disease and $53.8 \%$ had metastatic disease. The median number of prior systemic cancer therapies was 3 (range: 1-12). Descriptive characteristics for the overall study population and each DL are shown in table 1.

\section{Toxicity}

No DLTs were observed in this study. Right upper quadrant abdominal pain in a patient with liver metastases was the only grade 3 treatment-related $\mathrm{AE}$; all others were grade 1 or 2. Notably, four patients (31\%) experienced grade 1 and 2 CRS with a dose-effect trend, supported by the correlation between frequency and grade of AEs with DL. While fever, chills/rigor, and hypotension were 
Table 1 Baseline characteristics and demographic data of enrolled patients presented by dose level

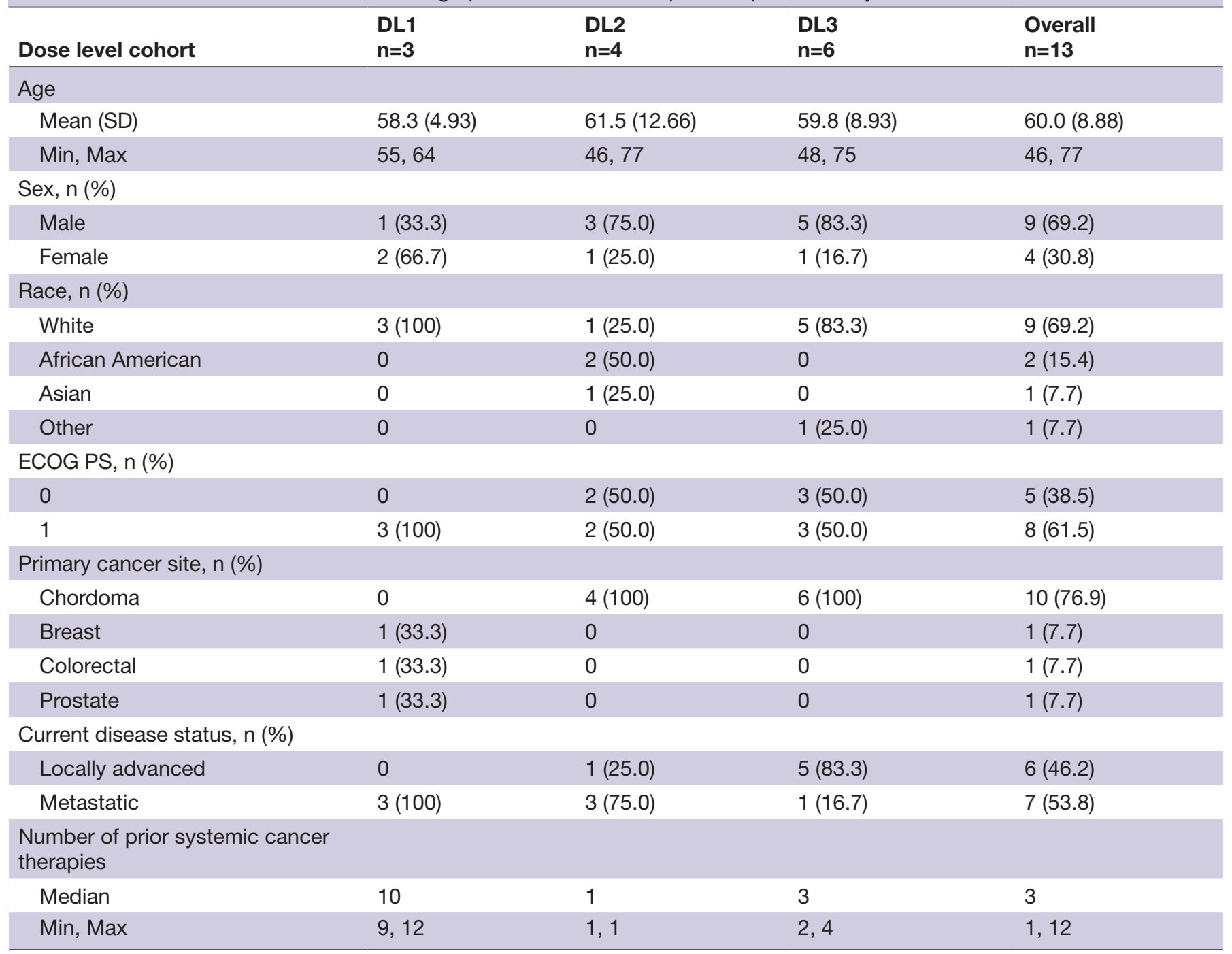

DL, dose level [one semicolon, not two]; ; ECOG PS, Eastern Cooperative Oncology Group Performance Status.

the most frequent manifestations across all DLs, these AEs were observed in $100 \%$ of patients treated at DL3. Observed symptoms of CRS across all DLs included fever $(69 \%)$, chills $(69 \%)$, hypotension $(62 \%)$, fatigue $(62 \%)$, headache $(46 \%)$, nausea $(39 \%)$, and myalgia $(39 \%)$, among others. Changes in temperature and blood pressure associated with CRS were transient across DLs and returned to normal levels mostly within 24 hours. Two patients were treated with glucocorticoids and intravenous crystalloid volume resuscitation and all recovered completely. Chills were subjectively intense but improved with meperidine. The nausea/emesis reported is thought to be attributed to meperidine due to temporal association following meperidine administration. AEs categorized as clinical signs and symptoms possibly related to CRS are summarized in table 2.

CRS typically manifested 6-8 hours after vaccine administration, peaked at 8-12 hours, and resolved within 24 hours. The timing of the most frequent manifestations of CRS and its relationship with DLs is shown in figure 2. Of note, elevated $\mathrm{C}$ reactive protein (CRP) was observed within 24 hours post-vaccination and returned to baseline over a similar time course as the development and resolution of CRS-related AEs. No elevation of CRP was observed in DL1, only a 1.4-fold increase in DL2 and 8.2-fold increase in DL3 (online supplemental figure 1). No signs or signals of cardiac, hepatic, pulmonary, renal, or bone marrow toxicity were detected. Other than CRS, AEs were uncommon and considered unrelated to the investigational drug. For example, one case of grade 2 hyponatremia, a serious AE, was attributed to syndrome of inappropriate antidiuretic hormone secondary to disease progression in a case of clival chordoma. One case of grade 2 bullous pemphigoid requiring corticosteroid therapy was attributed to prior immune checkpoint inhibitor (ICI) therapy (ie, antiprogrammed cell death 1 (PD-1) monoclonal antibody) due to the presence of baseline autoantibodies. Attribution of this immune-related $\mathrm{AE}$ was confounded by the antecedent development of infection with SARS-CoV-2. A detailed description of this case was previously reported. ${ }^{35}$ 
Table 2 Adverse events

\begin{tabular}{|c|c|c|c|c|c|c|c|c|c|c|}
\hline \multirow[b]{2}{*}{ Symptom } & \multirow{2}{*}{$\begin{array}{l}\text { Overall, } \\
n=13 \text { pts. } \\
n(\%)\end{array}$} & \multicolumn{3}{|l|}{$\begin{array}{l}\text { Cohort DL1, } \\
\mathrm{n}=3 \text { pts. }\end{array}$} & \multicolumn{3}{|l|}{$\begin{array}{l}\text { Cohort DL2, } \\
\mathrm{n}=4 \text { pts. }\end{array}$} & \multicolumn{3}{|l|}{$\begin{array}{l}\text { Cohort DL3, } \\
\mathrm{n}=6 \text { pts. }\end{array}$} \\
\hline & & Grades $1 \& 2$ & G 1 & G 2 & Grades $1 \& 2$ & G 1 & G 2 & Grades $1 \& 2$ & G 1 & G 2 \\
\hline Chills/rigor & $9(69.2)$ & $2(66 \%)$ & 2 & & $1(25 \%)$ & 1 & & $6(100 \%)$ & 2 & 4 \\
\hline Hypotension & $8(61.5)$ & & & & $2(50 \%)$ & 2 & & $6(100 \%)$ & 4 & 2 \\
\hline Nausea & $5(38.5)$ & $2(66 \%)$ & 2 & & & & & $3(50 \%)$ & 3 & \\
\hline Myalgia & 5 (38.5) & $1(33 \%)$ & & 1 & $2(50 \%)$ & 2 & & $2(33 \%)$ & 2 & \\
\hline Vomiting & $4(30.8)$ & & & & $1(25 \%)$ & 1 & & $3(50 \%)$ & 2 & 1 \\
\hline Dizziness & $4(30.8)$ & & & & $1(25 \%)$ & 1 & & $3(50 \%)$ & 3 & \\
\hline Anorexia & $1(7.7)$ & & & & $1(25 \%)$ & 1 & & & & \\
\hline $\begin{array}{l}\text { Concentration } \\
\text { impairment }\end{array}$ & $1(7.7)$ & & & & & & & $1(16 \%)$ & 1 & \\
\hline Delirium & $1(7.7)$ & & & & & & & $1(16 \%)$ & 1 & \\
\hline Itching & $1(7.7)$ & & & & & & & $1(16 \%)$ & 1 & \\
\hline Neuropathy & $1(7.7)$ & & & & & & & $1(16 \%)$ & 1 & \\
\hline Rash in arms & $1(7.7)$ & & & & & & & $1(16 \%)$ & 1 & \\
\hline Any symptoms & $11(84.6)$ & & & & & & & & & \\
\hline
\end{tabular}

Adverse events considered at least potentially related to cytokine release syndrome summarized by subject and grade of severity. $\mathrm{DL}$, dose level; G, grade

\section{Immune analyses}

The Fluorescence-activated cell sorting (FACS) -based assay for $\mathrm{T}$ cells expressing the type I cytokines IFN- $\gamma$, TNF- $\alpha$, IL-2, and/or the degranulation marker CD107a following stimulation with overlapping peptide pools is decribed in detail in the Materials and Methods section. Representative flow cytometery plots of brachyury-specific $\mathrm{CD} 4^{+}$and $\mathrm{CD} 8^{+} \mathrm{T}$ cells developed in a patient after vaccination are shown in online supplemental figure 2. Preexisting brachyury, CEA, and MUC1-specific T cells were detected in $54 \%, 33 \%$, and $73 \%$ of patients, respectively (online supplemental figure 3). The presence of preexisting $\mathrm{T}$ cell responses at baseline did not correspond with the development of TAA responses during therapy. Including all DLs and all time points examined, 9/13 (69\%) patients developed $\mathrm{CD}^{+}$and/or $\mathrm{CD}^{+} \mathrm{T}$ cell responses after vaccination to brachyury, the antigen encoded by the vaccine, while $7 / 8(88 \%)$ and $7 / 11(64 \%)$ developed $\mathrm{CD}^{+}$and/or $\mathrm{CD}^{+} \mathrm{T}$ cell responses to the 'cascade' antigens CEA and MUC1 not encoded in the vaccine (online supplemental figure 4). The induction of antigen-specific $\mathrm{T}$ cells by MVA-BN-brachyury-TRICOM given intravenously was rapid, with the majority of patients $(75 \%)$ having an increase in $\mathrm{CD} 4^{+}$and/or $\mathrm{CD}^{+}$ $\mathrm{T}$ cells targeting at least one of the antigens tested after a single dose of vaccine. Polyfunctional TAA responses, defined as $\mathrm{CD} 4^{+}$or $\mathrm{CD} 8^{+} \mathrm{T}$ cells that express two or more of the markers IFN- $\gamma$, TNF- $\alpha$, IL-2, or CD107a, were also measured before and after vaccination. Using the criteria of a $>3$-fold increase post- versus pre-vaccination, or the presence of $>100$ polyfunctional cells post-vaccination per $1 \times 10^{6}$ PBMCs (if negative at pre), polyfunctional $\mathrm{T}$ cells specific for brachyury, CEA, and MUC1 were generated in $62 \%, 75 \%$, and $45 \%$ of patients, respectively (figure 3 ). There was a trend of a dose-related increase in the development of multifunctional brachyury-specific $\mathrm{T}$ cells noted, with $1 / 3(33 \%)$ patients developing brachyuryspecific T cells at DL1, 2/4 (50\%) at DL2, and 5/6 (83\%) at DL3. The magnitude of polyfunctional $\mathrm{CD}^{+}$and $\mathrm{CD}^{+}$ $T$ cells generated in each patient after (vs before) vaccination can be seen in online supplemental figure 5 .

\section{Clinical outcomes}

An end-of-study evaluation of ORR (3 months after the last vaccination) in April 2021 per RECIST 1.1 showed one patient with a partial response (PR), four with stable disease (SD), and eight with progressive disease. No complete responses were observed. One patient with a PR and three with SD experienced clinical benefit with symptomatic improvement in pain and decreased use of daily 


\section{(Mean) Vital Signs Within 48 Hours After Vaccination}

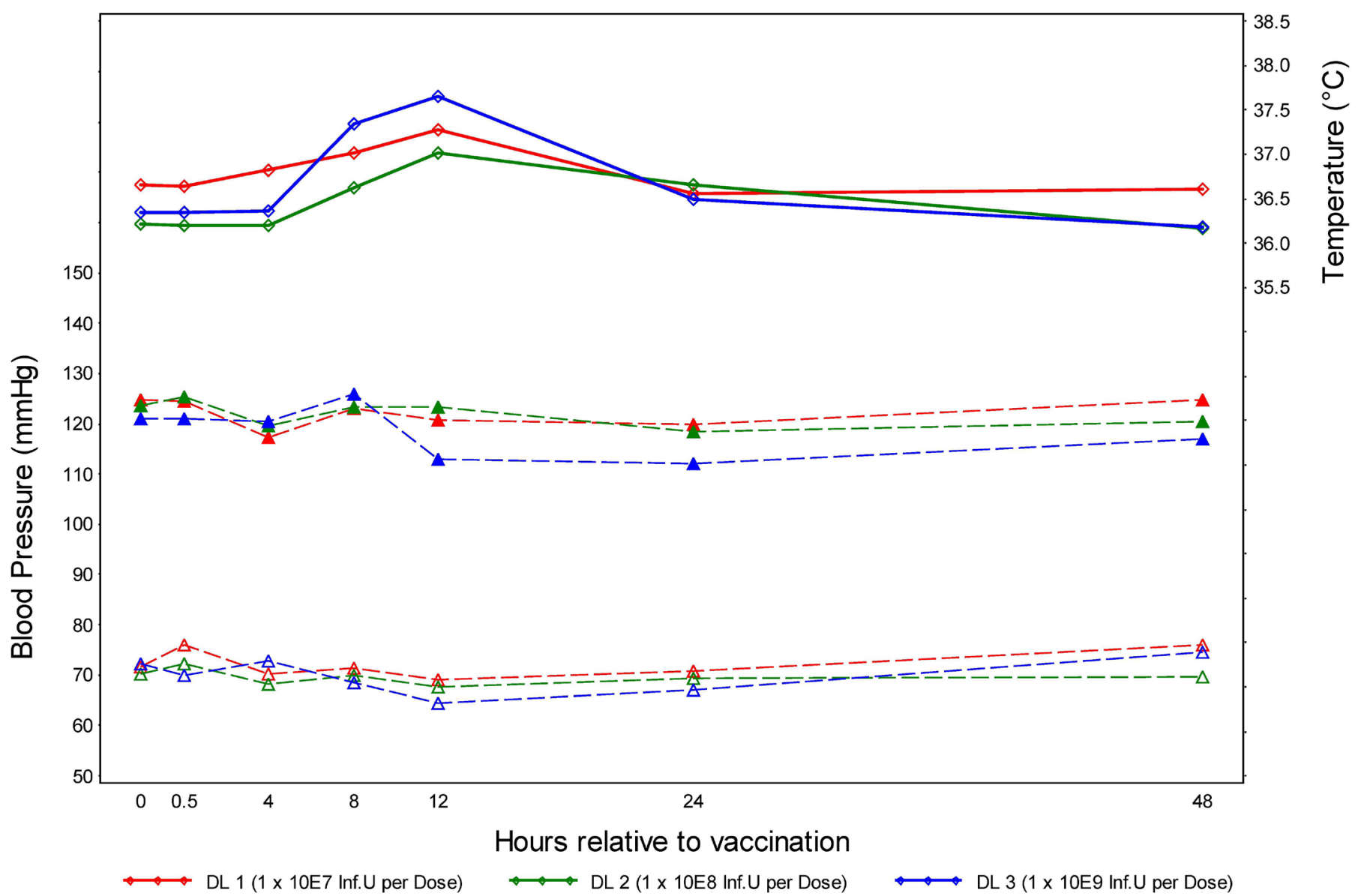

Figure 2 Change in vital signs within 48 hours post-vaccination. A transient increase in temperature and decrease in blood pressure were observed with a dose-effect trend. DL, dose level; Inf. U., infectious units.

analgesics. Exploratory retrospective response assessment using volumetric analysis performed by an experienced, blinded radiologist showed that while only one PR was observed per RECIST 1.1, two volumetric responses were observed. Patient \#9, whose tumor decreased by only $33 \%$ per RECIST 1.1, had a $66 \%$ shrinkage in tumor volume per volumetric analysis. Patient \#12 had a minimal decrease in tumor length of $2.7 \%$ per RECIST 1.1, which was considered SD; however, volumetric analysis showed a $41 \%$ decrease in tumor volume. This patient also had significant pain reduction, evidenced by decreasing pain scores and reduced opioid requirement. Of note, retrospective volumetric analysis was limited to patients with chordoma only. Efficacy results per RECIST 1.1 and volumetric analysis are summarized in table 3 .

\section{Case report of partial responder}

One PR per RECIST 1.1 was observed in patient \#9, a 59-year-old Caucasian man with metastatic chordoma of the lumbar spine extending through the anterior abdomen (figure 4). During 5 years of prior treatments, this chordoma failed to respond to surgical resection, proton radiation therapy, cryoablation, nivolumab, and other investigational therapies including NKTR-214, also known as bempegalgesleukin, a CD122-preferential IL-2 pathway agonist. The patient did not receive any therapy over the 6-month period prior to treatment on study. He received three treatments with intravenous MVA-BNbrachyury-TRICOM at DL3 over a 2-month treatment window. In the 48 hours following his first treatment, the patient noted a sensation of fullness in his abdomen as if his tumor were swollen. He developed concomitant edema, likely secondary to an inflammatory response in the tumor causing compression of the lower extremity vasculature, which resolved within a week. Prior to the third vaccine treatment, it was readily apparent on exam that the previously exophytic tumor was regressing. Areas of skin breakdown that had previously oozed blood had begun to heal. Clinical improvements in pain were also noted, both in terms of decreasing subjective pain scores as well as daily requirements of opioid analgesia. By the end-of-treatment visit, his skin had completely healed and only a small piece of residual tumor remained visible outside of the abdomen. End-of-treatment response assessment showed a 33\% decrease in tumor size per RECIST 1.1 but a more impressive $66 \%$ decrease in tumor volume. Prior to vaccination, this patient had detectable levels of peripheral $\mathrm{CD} 4^{+} \mathrm{T}$ cells specific for brachyury (online supplemental figure 3), and after 
Multifunctional TAA Responses by \# of Vaccines

\begin{tabular}{|c|c|c|c|c|c|c|c|c|c|c|}
\hline \multirow[b]{2}{*}{ PT } & \multirow[b]{2}{*}{ BOR } & \multirow[b]{2}{*}{ DL } & \multirow{2}{*}{$\begin{array}{c}\text { Timepoint } \\
\text { Day } \\
\text { (vs pre) }\end{array}$} & \multirow{2}{*}{$\begin{array}{l}\text { Post \# } \\
\text { Vaccines } \\
\text { (vs pre) }\end{array}$} & \multicolumn{2}{|c|}{ Brachyury } & \multicolumn{2}{|c|}{ CEA } & \multicolumn{2}{|c|}{ MUC1 } \\
\hline & & & & & CD4 2+ & CD8 2+ & CD4 2+ & CD8 2+ & CD4 2+ & CD8 2+ \\
\hline PT2 & PD & \multirow{4}{*}{1} & D22 & 1 & & & & ++ & & \\
\hline PT2 & PD & & D43 & 2 & + & + & & + & + & \\
\hline PT3 & PD & & D22 & 1 & & & & & & \\
\hline PT4 & $\mathrm{PD}$ & & D42 & 2 & & & ++ & & & \\
\hline PT5 & PD & \multirow{9}{*}{2} & D22 & 1 & + & + & & & & \\
\hline PT6 & PD & & D22 & 1 & & & & & & \\
\hline PT6 & PD & & D113 & $2^{\gamma}$ & & & & & & \\
\hline PT7 & $\mathrm{SD}^{\alpha}$ & & D113 & $1^{\theta}$ & & & & & & \\
\hline PT7 & $\mathrm{SD}^{\alpha}$ & & D134 & 2 & & & & & & \\
\hline PT7 & $\mathrm{SD}^{\alpha}$ & & D161 & 3 & & & & & & \\
\hline PT8 & SD & & D22 & 1 & \multirow{3}{*}{++} & \multirow[t]{3}{*}{+} & \multirow{3}{*}{++} & \multirow{3}{*}{$\begin{array}{l}++ \\
+\end{array}$} & \multirow{3}{*}{\multicolumn{2}{|c|}{++}} \\
\hline PT8 & SD & & D43 & 2 & & & & & & \\
\hline PT8 & SD & & D70 & 3 & & & & & & \\
\hline PT9 & $\mathrm{PR}^{\alpha}$ & \multirow{17}{*}{3} & D22 & 1 & & + & & & & \\
\hline PT9 & $\mathbf{P R}^{\alpha}$ & & D70 & 3 & & & & & & \\
\hline PT10 & PD & & D22 & 1 & & & & & & \\
\hline PT10 & PD & & D43 & 2 & & & & & & \\
\hline PT10 & PD & & D72 & 3 & & ++ & & & ++ & ++ \\
\hline PT11 & PD & & D22 & 1 & \multirow{3}{*}{\multicolumn{2}{|c|}{+}} & & + & & + \\
\hline PT11 & PD & & D43 & 2 & & & & & & \\
\hline PT11 & PD & & D70 & 3 & & & & & ++ & + \\
\hline PT12 & $\mathrm{SD}^{\alpha}$ & & D22 & 1 & \multirow{3}{*}{++} & \multirow{3}{*}{+} & & & \multirow{3}{*}{\multicolumn{2}{|c|}{++}} \\
\hline PT12 & $\mathrm{SD}^{\alpha}$ & & D43 & 2 & & & + & ++ & & \\
\hline PT12 & $\mathrm{SD}^{\alpha}$ & & D70 & 3 & & & + & ++ & & \\
\hline PT13 & $\mathrm{SD}^{\alpha}$ & & D22 & 1 & & & \multirow{3}{*}{\multicolumn{2}{|c|}{+}} & & \\
\hline PT13 & $\mathrm{SD}^{\alpha}$ & & D43 & 2 & & & & & & \\
\hline PT13 & $\mathrm{SD}^{\alpha}$ & & D70 & 3 & & & & & & \\
\hline PT14 & PD & & D22 & 1 & \multirow{3}{*}{$\begin{array}{l}++ \\
+\end{array}$} & \multirow{3}{*}{$\begin{array}{l}++ \\
+\end{array}$} & & & & \\
\hline PT14 & PD & & D43 & 2 & & & & & & \\
\hline PT14 & PD & & D70 & 3 & & & & & & \\
\hline
\end{tabular}

Figure 3 Development of multifunctional antigen specific $C D 4^{+}$and $C D 8^{+} T$ cell responses post- (vs pre-) MVA-BN-brachyuryTRICOM vaccine. TAA responses againstbrachyury and the cascade antigens CEA and MUC1 were compared in thirteen patients pre- and post- 1,2 , and 3 vaccinations, where sufficient research bloods were available. The absolute number of multifunctional TAA responses (CD4 ${ }^{+}$or $\mathrm{CD}^{+}$T cells expressing two or more of the following: IFN- $\gamma$, TNF- $\alpha$, IL-2, or CD107a) per $1 \times 10^{6}$ PBMCs plated at the start of the stimulation assay was calculated. Any background signal obtained with the HLA peptide pool was subtracted. Patients were scored as having a $>3$-fold (or if no cells at pre, $>100 / 1 \times 10^{6}$ cells at post, + ) or $>10$ fold (or if no cells at pre, $>1000 / 1 \times 10^{6}$ cells at post, ++ ) increase in $C D 4^{+}$or $C D 8^{+}$multifunctional cells post- (vs pre) vaccine. Gray indicates where an insufficient number of viable PBMCs were recovered for analysis. BOR, best overall response; CEA, carcinoembryonic antigen; MUC, mucin; PD, progressive disease; PR, partial response; PT, patient; SD, stable disease; TAA, tumor-associated antigen.

one vaccine dose (D22), displayed a transient increase in $\mathrm{CD}^{+} \mathrm{T}$ cells specific for brachyury that were characterized by the production of IFN- $\gamma$ and TNF- $\alpha$ (online supplemental figure 4). Flow cytometry assessment of the degree of immune infiltrate in a biopsy taken 2 days after the third vaccine in this patient showed that $71 \%$ of viable cells were leukocytes $\left(\mathrm{CD} 45^{+}\right)$. Among the leukocytes, $39 \%$ were $\mathrm{CD} 4^{+} \mathrm{T}$ cells and $31 \%$ were $\mathrm{CD} 8^{+} \mathrm{T}$ cells. This percentage of $\mathrm{T}$ cells equates to approximately 2466 CD $4^{+} \mathrm{T}$ cells $/ \mathrm{cm}^{2}$ and $1966 \mathrm{CD} 8^{+} \mathrm{T}$ cells $/ \mathrm{cm}^{2}$ in the biopsy sample obtained. Within the $\mathrm{CD} 4^{+}$and $\mathrm{CD} 8^{+}$ $\mathrm{T}$ cell compartments, the majority of cells detected were either a central memory $\left(\mathrm{CCR} 7^{+}, \mathrm{CD} 45 \mathrm{RA}^{-}\right)$or effector memory (CCR7 $7^{-} \mathrm{CD}^{2} 5 \mathrm{RA}^{-}$) phenotype; $47 \%$ of $\mathrm{CD} 4^{+} \mathrm{T}$ cells and $24 \%$ of $\mathrm{CD}^{+} \mathrm{T}$ cells were central memory cells, while $52 \%$ of $\mathrm{CD}^{+} \mathrm{T}$ cells and $75 \%$ of $\mathrm{CD}^{+} \mathrm{T}$ cells were effector memory cells. Prior to enrolling in this study, the patient was in clinical decline and considering transitioning to hospice care to focus on palliation of pain. As of the writing of this article, the patient's tumor has not 
Table 3 Clinical efficacy (ORR per RECIST 1.1) and volumetric analysis

\begin{tabular}{|c|c|c|c|c|}
\hline Dose level & Patient no. & Pain improvement & $\begin{array}{l}\text { RECIST at the last assessment ( } 3 \\
\text { months after first dose) }\end{array}$ & $\begin{array}{l}\text { Volumetric change, \% change } \\
\text { (baseline/study end; } \mathrm{cm}^{3} \text { ) }\end{array}$ \\
\hline \multirow[t]{2}{*}{1} & 2 & No & PD (clin. deterioration) & Not done \\
\hline & 4 & No & PD (clin. deterioration) & Not done \\
\hline \multirow[t]{3}{*}{2} & 5 & No & PD & $+100(15.3 / 30)$ \\
\hline & 6 & No & PD & $+63.5(7.4 / 12.1)$ \\
\hline & 8 & No & SD & Not done (too small to measure) \\
\hline \multirow[t]{3}{*}{3} & 9 & Yes & PR & $-66(2146 / 724)$ \\
\hline & 10 & No & PD & $+75(0.8 / 1.4)$ \\
\hline & 11 & No & PD & +212(10.7/33.4) \\
\hline $\begin{array}{l}\text { All dose } \\
\text { levels }\end{array}$ & $n=13$ & $\begin{array}{l}\text { Yes } 4 \text { out of } 13 \\
\text { No } 9 \text { out of } 13\end{array}$ & $\begin{array}{l}1 \mathrm{PR} \\
4 \mathrm{SD} \\
8 \mathrm{PD}\end{array}$ & Two with volumetric shrinkage \\
\hline
\end{tabular}

Summary of clinical efficacy results in all patients and per dose level, with a comparison of ORR per RECIST 1.1 and retrospective exploratory volumetric analysis.

ORR, objective response rate; PD, progressive disease; PR, partial response; RECIST, Response Evaluation Criteria in Solid Tumors; SD; stable disease

grown (PR lasting $>10$ months) and the improvements in his performance status and quality of life remain durable (online supplemental figure 5)

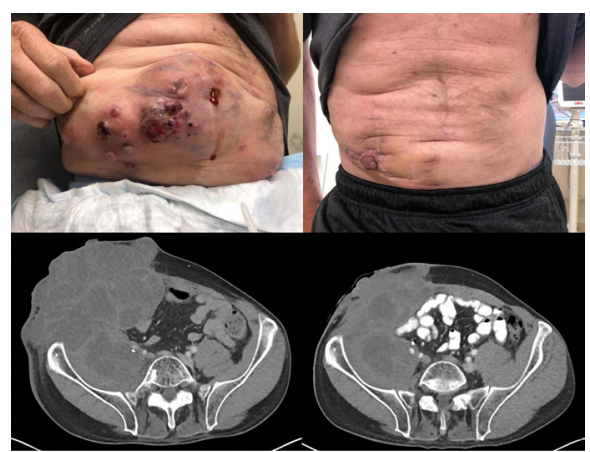

Figure 4 Patient \#9 (responder). Before and after treatment photos and CT imaging for patient \#9, a 59-year-old man with metastatic chordoma of the lumbar spine extending through the anterior abdomen. He had previously failed multiple lines of treatment. Following three doses of the investigational intravenous brachyury-targeting vaccine (DL3) given over a 2-month treatment window, the patient's tumor shrank $33 \%$ per RECIST $1.1 \%$ and $66 \%$ per exploratory volumetric analysis. Left top: clinic photograph from August 14, 2020, prior to first dose of DL3 vaccine. Left bottom: baseline CT from August 14, 2020. Right top: clinic photograph from October 27, 2020, after three vaccine doses. Right bottom: End-of-treatment CT from October 27, 2020. DL, dose level; RECIST, Response Evaluation Criteria in Solid Tumors.

\section{DISCUSSION}

From January 2020 to March 2021, 13 patients were treated with three DLs of intravenous MVA-BN-brachyuryTRICOM vaccine. The maximum tolerated dose was not reached. The maximum administered dose was $10^{9}$ Inf. U. every 3 weeks for three doses, which was safe and well tolerated with no dose reductions or discontinuations due to safety. No serious AEs were attributed to the vaccine. Vaccine-related toxicities were consistent with class profile (ie, influenza-like symptoms). CRS up to grade 2 was observed with no adverse outcomes. A dose-effect trend was observed for fever, chills/rigor, and hypotension. One PR at DL3 (7.7\%) was observed, a 33\% decrease in tumor size per RECIST measurement, and 66\% decrease per exploratory volumetric measurement. While no validated volumetric response criteria exist for chordoma, exploratory analysis performed by a blinded radiologist showed volumetric response in a second patient with chordoma who also reported significant reduction in his pain level. Additionally, three patients $(31 \%)$ experienced clinical benefit in the form of decreased pain $(50 \%$ of patients at DL3). Immune correlative studies show that intravenous administration of MVA-BN-brachyury-TRICOM generated brachyury-specific $\mathrm{T}$ cells in most patients demonstrating the immunogenicity of this vaccine. In addition, $\mathrm{T}$ cell responses against CEA and MUC1, antigens not encoded in the vaccine, were induced after vaccination, suggesting that MVA-BN-brachyury-TRICOM generates immunologically relevant tumor-cell destruction. Others have shown that long-lasting polyfunctional T cells are associated with 
improved overall survival and can be induced by vaccination. ${ }^{36}$ In the current study, the majority of patients generated polyfunctional $\mathrm{T}$ cell responses to brachyury as well as against cascade antigens. Of note, we observed a trend indicating a potential dose-related response in the generation of multifunctional brachyury-specific $\mathrm{T}$ cells. After consideration of safety data and immune activation, the RP2D for intravenous administration of MVABN-brachyury-TRICOM was determined to be $10^{9} \mathrm{Inf}$. U. $\mathrm{q} 3 \mathrm{w} \times 3$ doses.

While this study was not powered to demonstrate efficacy, the single PR in a patient with advanced sacral chordoma was compelling. Not only was the response impressive on clinical exam and CT imaging, but the patient also experienced a meaningful and durable improvement in quality of life. It remains unclear what personal or disease factors may have contributed to this patient's profound response on study. Significant skin breakdown was noted at study baseline, and it is possible that the immune system was primed in similar fashion to the original immunotherapy responders of Coley ${ }^{37}$ One must also consider that the patient was pretreated with more than one prior immunotherapy, including an anti-PD-1 ICI and investigational IL-2 agonist. Notably, the second patient who developed a response per exploratory volumetric analysis was also pre-treated with an ICI and derived significant clinical benefit. Both patients had ongoing responses as of June 1,2021 . It should be noted that the peripheral brachyuryspecific $T$ cells induced in the single patient developing a PR were transient. This phenomenon has been noted in numerous cancer vaccine trials ${ }^{25}$ 38-41 $^{3}$ and may reflect $\mathrm{T}$ cell trafficking into and out of the tumor as well as homeostatic regulation to suppress an immune response generated against self-antigens.

As the field of cancer immunotherapy continues to develop, vaccination remains a conceptually appealing strategy to effect a targeted immune response in patients with advanced malignancies. Yet there has been limited success to date in bringing therapeutic cancer vaccines or other viral immunotherapy products to market. ${ }^{42}$ The development of a clinically effective therapeutic cancer vaccine requires careful consideration of disease-specific factors (ie, the tumor model and appropriate selection of targetable TAAs) and other strategies to optimize immune response. In chordoma, brachyury remains the most promising TAA for the development of novel targeted therapies. While limited clinical trials of brachyurytargeting cancer vaccines have yet to demonstrate efficacy, this phase 1 study is unique as the first intravenous administration of a brachyury-targeting vaccine and the second intravenous administration of any cancer vaccine.$^{243}$ The immunogenicity induced by intravenous administration of MVA-BN-brachyury-TRICOM in the current study is comparable to that obtained with SC administration of MVA-brachyury-TRICOM vaccine in the phase 1 dosefinding study and a second phase 1 study employing MVA-brachyury-TRICOM as the priming vaccine and FPV-brachyury-TRICOM as the booster vaccine..$^{25} 26$ This may be due to the small number of patients evaluated, the heterogenicity of patients enrolled in these studies, and the varied time points in which research bloods were evaluated for immune assessments. Optimism for the potential increased efficacy of intravenous vaccine administration in cancers is supported by evidence from infectious disease studies showing the superior ability of intravenouslyadministered bacillus Calmette-Guérin to prevent Mycobacterium tuberculosis infection in non-human primates. ${ }^{44}$

In the future, researchers involved in the development of intravenouslyadministered MVA-BN-brachyuryTRICOM must consider not only appropriate clinical endpoints, but whether it is appropriate to pursue a phase 2 efficacy study in chordoma. While ORR per RECIST 1.1 remains a valid surrogate to demonstrate efficacy in single-arm clinical trials, volumetric chordomaspecific response criteria supported by patient-reported outcome data may prove an acceptable alternative. As responses (per RECIST 1.1 and volumetric analysis) observed in our study were limited to patients with spinal chordomas, future efficacy trials may consider excluding patients with clival lesions due to potential for increased intracranial pressure from vaccine-mediated inflammation. It is unclear if tumor biology differs between clival and sacral chordomas to the extent that the tumors should be classified separately. Clival lesions are not only more difficult to measure due to their small size, but the potential for increased intracranial pressure from vaccine-mediated inflammation could present a risk to these patients.

If a phase 2 study of a single-agent vaccine in chordoma is not the next step in the development of treatments for this disease, what should be the goal of future phase 1 studies? Given that CRP levels rose to their highest level following administration of the first vaccine dose, fewer doses may be explored. Given the safety of intravenouslyadministered MVA-BN-brachyury-TRICOM and the low grade of CRS observed, hospitalizations may be shorter in future studies. Finally, since both responding patients (by RECIST 1.1 and volumetric analysis) were previously treated with an ICI, is immune priming necessary to generate a clinical response? Future combination studies will evaluate potential synergies between this intravenouslyadministered brachyury-targeting vaccine and other immunotherapeutic cancer drugs. Appropriate clinical endpoints will be important. While ORR per RECIST 1.1 remains a valid surrogate to demonstrate efficacy in single-arm clinical trials, volumetric chordoma-specific response criteria supported by patient-reported outcome data could be an acceptable alternative. Future studies will evaluate potential synergies between this intravenouslyadministered brachyury-targeting vaccine and other immuno-oncology drugs. Given the rarity of chordoma and the paucity of patients available and eligible to enroll on study, careful consideration of study design is paramount. 


\section{Author affiliations}

${ }^{1}$ Genitourinary Malignancies Branch, National Cancer Institute, National Institutes of Health, Bethesda, Maryland, USA

${ }^{2}$ Medical Oncology Service, Center for Cancer Research, National Cancer Institute, National Institutes of Health, Bethesda, Maryland, USA

${ }^{3}$ Laboratory of Tumor Immunology and Biology, National Cancer Institute, Bethesda, Maryland, USA

${ }^{4}$ Pediatric Oncology Branch, National Cancer Institute, Bethesda, Maryland, USA

${ }^{5}$ Oncology Strategy, Bavarian Nordic Inc, Morrisville, North Carolina, USA

${ }^{6}$ Bavarian Nordic GmbH, Martinsried, Bayern, Germany

${ }^{7}$ Arcellix, Inc, Gaithersburg, Maryland, USA

Twitter Ravi A Madan @Dr_RaviMadan, Charalampos Floudas @chfloudas, Jason Mark Redman @theredmanMD, James L Gulley @gulleyj1 and Marijo Bilusic @ mbilusic

Acknowledgements We acknowledge and thank the patients, associated study staff, and sponsor for their support of this study. The authors thank Ariana Sabzevari and Keanan Wright for technical assistance with immune assays. The authors thank Bonnie L. Casey for editorial assistance in the preparation of this manuscript.

Contributors TA, CP-N, CH, JS, JLG, MB contributed to conception/design. PJDM, $\mathrm{KL}-\mathrm{W}, \mathrm{RAM}, \mathrm{FK}, \mathrm{CF}, \mathrm{JS}$, JLM, JMR, JLG, MB contributed to provision of study material or patients. PJDM, KL-W, RAM, FK, RND, AS, CP, CJ, CF, JS, JLM, JMR, JS, JLG, MB contributed to collection and/or assembly of data. PJDM, RND, AS, CP, $\mathrm{CJ}, \mathrm{BK}, \mathrm{CP}-\mathrm{N}, \mathrm{ED}, \mathrm{BW}, \mathrm{JS}, \mathrm{JLG}, \mathrm{MB}$ contributed to data analysis and interpretation. PJDM, RND, MB contributed to manuscript writing. All authors contributed to final approval of manuscript.

Funding This research was supported by the Intramural Research Program of the Center for Cancer Research, National Cancer Institute, National Institutes of Health and a cooperative research and development agreement between the National Cancer Institute and Bavarian Nordic.

Competing interests BK: Bavarian Nordic (E). TA: Bavarian Nordic (E). CP-N: Bavarian Nordic (E). CH: Arcellx (E) (previously employed by Bavarian Nordic). The other authors indicate no financial relationships. (E) Employment.

\section{Patient consent for publication Not required.}

Ethics approval The study protocol was approved by the Institutional Review Board of the Center for Cancer Research, National Cancer Institute. The study was conducted according to the principles of the Declaration of Helsinki and was performed in compliance with Good Clinical Practice guidelines. Written informed consent was obtained from each patient.

Provenance and peer review Not commissioned; externally peer reviewed.

Data availability statement Data are available upon reasonable request.

Supplemental material This content has been supplied by the author(s). It has not been vetted by BMJ Publishing Group Limited (BMJ) and may not have been peer-reviewed. Any opinions or recommendations discussed are solely those of the author(s) and are not endorsed by BMJ. BMJ disclaims all liability and responsibility arising from any reliance placed on the content. Where the content includes any translated material, BMJ does not warrant the accuracy and reliability of the translations (including but not limited to local regulations, clinical guidelines, terminology, drug names and drug dosages), and is not responsible for any error and/or omissions arising from translation and adaptation or otherwise.

Open access This is an open access article distributed in accordance with the Creative Commons Attribution Non Commercial (CC BY-NC 4.0) license, which permits others to distribute, remix, adapt, build upon this work non-commercially, and license their derivative works on different terms, provided the original work is properly cited, appropriate credit is given, any changes made indicated, and the use is non-commercial. See http://creativecommons.org/licenses/by-nc/4.0/.

\section{ORCID iDs}

Peter J DeMaria http://orcid.org/0000-0003-1313-6065

Ravi A Madan http://orcid.org/0000-0001-5106-8636

Fatima Karzai http://orcid.org/0000-0002-3244-1332

Claudia Palena http://orcid.org/0000-0002-0445-4486

Caroline Jochems http://orcid.org/0000-0002-9000-9855

Charalampos Floudas http://orcid.org/0000-0002-0020-237X

Jason Mark Redman http://orcid.org/0000-0001-8200-0167

Jeffrey Schlom http://orcid.org/0000-0001-7932-4072

James L Gulley http://orcid.org/0000-0002-6569-2912
Marijo Bilusic http://orcid.org/0000-0003-1020-689X

\section{REFERENCES}

1 Fernando RI, Castillo MD, Litzinger M, et al. II-8 signaling plays a critical role in the epithelial-mesenchymal transition of human carcinoma cells. Cancer Res 2011;71:5296-306.

2 Herrmann BG, Labeit S, Poustka A, et al. Cloning of the T gene required in mesoderm formation in the mouse. Nature 1990;343:617-22.

3 Edwards YH, Putt W, Lekoape KM, et al. The human homolog T of the mouse T(brachyury) gene; gene structure, cDNA sequence, and assignment to chromosome 6q27. Genome Res 1996;6:226-33.

4 Kispert A, Hermann BG. The Brachyury gene encodes a novel DNA binding protein. Embo $J$ 1993;12:4898-9.

5 Kispert A, Koschorz B, Herrmann BG. The T protein encoded by brachyury is a tissue-specific transcription factor. Embo $\mathrm{J}$ 1995;14:4763-72.

6 Palena C, Polev DE, Tsang KY, et al. The human T-box mesodermal transcription factor brachyury is a candidate target for T-cellmediated cancer immunotherapy. Clin Cancer Res 2007;13:2471-8.

7 Kispert A, Herrmann BG, Leptin M, et al. Homologs of the mouse brachyury gene are involved in the specification of posterior terminal structures in Drosophila, Tribolium, and Locusta. Genes Dev 1994;8:2137-50.

8 Wilkinson DG, Bhatt S, Herrmann BG. Expression pattern of the mouse $\mathrm{T}$ gene and its role in mesoderm formation. Nature 1990;343:657-9.

9 Schulte-Merker S, Smith JC. Mesoderm formation in response to brachyury requires FGF signalling. Curr Biol 1995;5:62-7.

10 Yamaguchi TP, Takada S, Yoshikawa Y, et al. T (Brachyury) is a direct target of Wnt3a during paraxial mesoderm specification. Genes Dev 1999;13:3185-90.

11 Roselli M, Fernando RI, Guadagni F, et al. Brachyury, a driver of the epithelial-mesenchymal transition, is overexpressed in human lung tumors: an opportunity for novel interventions against lung cancer. Clin Cancer Res 2012;18:3868-79.

12 Richardson SM, Ludwinski FE, Gnanalingham KK, et al. Notochordal and nucleus pulposus marker expression is maintained by subpopulations of adult human nucleus pulposus cells through aging and degeneration. Sci Rep 2017;7:1501.

13 Thiery JP, Sleeman JP. Complex networks orchestrate epithelialmesenchymal transitions. Nat Rev Mol Cell Biol 2006;7:131-42.

14 Kalluri R, Weinberg RA. The basics of epithelial-mesenchymal transition. J Clin Invest 2009;119:1420-8.

15 Gravdal K, Halvorsen OJ, Haukaas SA, et al. A switch from $\mathrm{E}$-cadherin to $\mathrm{N}$-cadherin expression indicates epithelial to mesenchymal transition and is of strong and independent importance for the progress of prostate cancer. Clin Cancer Res 2007;13:7003-11.

16 Onder TT, Gupta PB, Mani SA, et al. Loss of E-cadherin promotes metastasis via multiple downstream transcriptional pathways. Cancer Res 2008;68:3645-54.

17 Fernando RI, Litzinger M, Trono P, et al. The T-box transcription factor brachyury promotes epithelial-mesenchymal transition in human tumor cells. J Clin Invest 2010;120:533-44.

18 Vujovic S, Henderson S, Presneau N, et al. Brachyury, a crucial regulator of notochordal development, is a novel biomarker for chordomas. J Pathol 2006;209:157-65.

19 Tirabosco R, Mangham DC, Rosenberg AE, et al. Brachyury expression in extra-axial skeletal and soft tissue chordomas: a marker that distinguishes chordoma from mixed tumor/ myoepithelioma/parachordoma in soft tissue. Am J Surg Pathol 2008;32:572-80.

20 Yang XR, Ng D, Alcorta DA, et al. T (brachyury) gene duplication confers major susceptibility to familial chordoma. Nat Genet 2009;41:1176-8.

21 Sharifnia T, Wawer MJ, Chen T, et al. Small-molecule targeting of brachyury transcription factor addiction in chordoma. Nat Med 2019;25:292-300.

22 Sheppard HE, Dall'Agnese A, Park WD, et al. Targeted brachyury degradation disrupts a highly specific autoregulatory program controlling chordoma cell identity. Cell Rep Med 2021;2:100188.

23 Heery CR, Singh BH, Rauckhorst M, et al. Phase I trial of a yeast-based therapeutic cancer vaccine (GI-6301) targeting the transcription factor brachyury. Cancer Immunol Res 2015;3:1248-56.

24 DeMaria PJ, Bilusic M, Park DM, et al. Randomized, double-blind, placebo-controlled phase II study of yeast-brachyury vaccine (GI6301 ) in combination with standard-of-care radiotherapy in locally advanced, unresectable chordoma. Oncologist 2021;26:e847-58. 
25 Heery CR, Palena C, McMahon S, et al. Phase I study of a poxviral TRICOM-based vaccine directed against the transcription factor brachyury. Clin Cancer Res 2017;23:6833-45.

26 Collins JM, Donahue RN, Tsai Y-T, et al. Phase I trial of a modified vaccinia ankara priming vaccine followed by a fowlpox virus boosting vaccine modified to express brachyury and costimulatory molecules in advanced solid tumors. Oncologist 2020;25:560-1006.

27 Hanke T, Blanchard TJ, Schneider J, et al. Immunogenicities of intravenous and intramuscular administrations of modified vaccinia virus Ankara-based multi-CTL epitope vaccine for human immunodeficiency virus type 1 in mice. J Gen Virol 1998;79:83-90.

28 Shen X, Wong SBJ, Buck CB, et al. Direct priming and crosspriming contribute differentially to the induction of CD8+ CTL following exposure to vaccinia virus via different routes. J Immunol 2002;169:4222-9.

29 Lauterbach H, Kassub R, Pätzold J, et al. Immune requirements of post-exposure immunization with modified vaccinia Ankara of lethally infected mice. PLoS One 2010;5:e9659.

30 Eisenhauer EA, Therasse P, Bogaerts J, et al. New response evaluation criteria in solid tumours: revised RECIST guideline (version 1.1). Eur J Cancer 2009;45:228-47.

31 U.S. Department of Health and Human Services. Common terminology criteria for adverse events (CTCAE) version 5.0. Available: https://ctep.cancer.gov/protocoldevelopment/electronic applications/docs/CTCAE_v5_Quick_Reference_8.5x11.pdf [Accessed 17 May 2021].

32 Lee DW, Santomasso BD, Locke FL, et al. ASTCT consensus grading for cytokine release syndrome and neurologic toxicity associated with immune effector cells. Biol Blood Marrow Transplant 2019;25:625-38.

33 Heery CR, Singh BH, Rauckhorst M, et al. Phase I trial of a yeast-based therapeutic cancer vaccine (GI-6301) targeting the transcription factor brachyury. Cancer Immunol Res 2015;3:1248-56.

34 Donahue RN, Lepone LM, Grenga I, et al. Analyses of the peripheral immunome following multiple administrations of avelumab, a human
IgG1 anti-PD-L1 monoclonal antibody. J Immunother Cancer 2017;5:20.

35 Pastor DM, Lee-Wisdom K, Arai AE, et al. Fast clearance of the SARS-CoV-2 virus in a patient undergoing vaccine immunotherapy for metastatic chordoma: a case report. Front Oncol 2020;10:603248.

36 Wimmers F, Aarntzen EHJG, Duiveman-deBoer T, et al. Longlasting multifunctional $\mathrm{CD}^{+} \mathrm{T}$ cell responses in end-stage melanoma patients can be induced by dendritic cell vaccination. Oncoimmunology 2016;5:e1067745.

37 Coley WB. The treatment of malignant tumors by repeated inoculations of erysipelas. With a report of ten original cases. 1893 Clin Orthop Relat Res 1991:3-11.

38 Dillon PM, Petroni GR, Smolkin ME, et al. A pilot study of the immunogenicity of a 9-peptide breast cancer vaccine plus poly-ICLC in early stage breast cancer. J Immunother Cancer 2017;5:92.

39 Melssen MM, Petroni GR, Chianese-Bullock KA, et al. A multipeptide vaccine plus toll-like receptor agonists LPS or polyICLC in combination with incomplete Freund's adjuvant in melanoma patients. J Immunother Cancer 2019;7:163.

40 Engelhard $\mathrm{VH}$, Obeng RC, Cummings KL, et al. MHC-restricted phosphopeptide antigens: preclinical validation and first-in-humans clinical trial in participants with high-risk melanoma. J Immunother Cancer 2020;8:e000262.

41 Liu H, Zha Y, Choudhury N, et al. WT1 peptide vaccine in montanide in contrast to poly ICLC, is able to induce WT1-specific immune response with TCR clonal enrichment in myeloid leukemia. Exp Hematol Oncol 2018;7:1.

42 DeMaria PJ, Bilusic M. Cancer vaccines. Hematol Oncol Clin North Am 2019;33:199-214.

43 Sahin U, Oehm P, Derhovanessian E, et al. An RNA vaccine drives immunity in checkpoint-inhibitor-treated melanoma. Nature 2020;585:107-12.

44 Darrah PA, Zeppa JJ, Maiello P, et al. Prevention of tuberculosis in macaques after intravenous BCG immunization. Nature 2020;577:95-102. 